\title{
Maximum Allowable Delay Bound Estimation in Networked Control of Bounded Nonlinear Systems
}

\author{
As hraf F. Khalil ${ }^{1, *}$, Jihong Wang ${ }^{2}$ \\ ${ }^{1}$ School of Electrical, Electronic and Computer Engineering, University of Birmingham, Birmingham, B15 2TT, UK \\ ${ }^{2}$ School of En gineering, University of Warwick, Coventry, CV4 7AL, UK
}

\begin{abstract}
Networked Control Systems (NCSs) has been recognized as an area where theory is behind the development of technology. The defining feature of NCSs can be considered as the information is exchanged through a network among control system components. So the network induced time delay is inevitable in NCSs. The time delay may degrade the performance of control systems and even destabilize the systems if they are designed without considering the effects of the time delays properly. Once the structure of a NCS is confirmed, it is essential to identify what the maximum time delay is allowed for maintaining the system stability which, in turn, is also associated with the process of controller design. This paper proposes a new method for estimating the maximum allowable time delay in networked control systems with norm bounded nonlinearity. The relation between the maximum nonlinearity and the maximum allowable delay is studied using the proposed method, and it is found that increasing the maximum nonlinearity bound reduces the maximum allowable delay. Furthermore, increasing the time delay leads to shrink the domain of attraction. The results of the maximum allowable delay bound and the maximum nonlinearity are compared with some of the published results.
\end{abstract}

Keywo rds Networked Control System, Stability, Nonlinearity, Norm Bounded, Maximum Allowable Delay Bound

\section{Introduction}

The advances in communication and network technology, and the availability of high-speed computers have resulted in an increasing interest in Networked Control Systems (NCSs). This type of control systems can be defined as a control system where the control loop is closed through a real-time communication netwo rk[1]. The term "Networked Control Systems" first appeared in Gregory C. Walsh's article in 1998[2]. A typical organization of an NCS is shown in Figure 1. In Networked Control Systems, the reference input, plant output and control input are exchange d through a real-time communication network. The main advantages of NCSs are modularity, simplified wiring, low cost, reduced weight, decentralization of control, integrated diagnosis, simple installation, quick and easy for maintenan ce[3], flexible expandability (easy to add/remove sensors, actuators or controllers with low cost). NCSs are able to easily fuse global information to make intelligent decisions over large physical spaces.

As the control loop is closed through a communication network, the time delay and data dropout are unavoidable. This may degrade the performance of NCSs or even destabilize the system.

* Corresponding author:

ashrafkhalilg@yahoo.com(AshrafF. Khalil)

Published online at http://journal.sapub.org/control

Copyright (C) 2012 Scientific \& Academic Publishing. All Rights Reserved

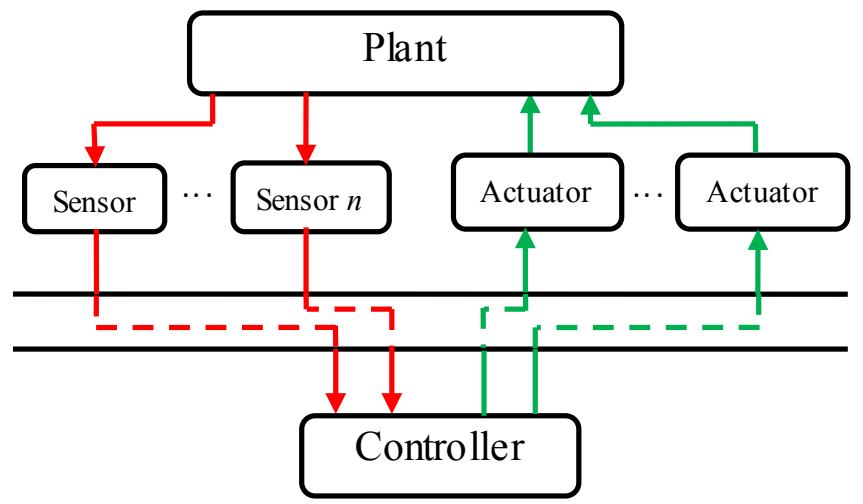

Figure 1. A Typical Networked System

In general, the control systems with time delays can be classified into time delay independent where the stability is not affected by the time delay and time delay dependent where the time delay affects the stability[4]. Time delay, no doubt, increases complexity in analysis and design of NCSs. Conventional control theories built on a number of standing assumptions; including synchronized control and non delayed sensing and actuation must be re-evaluated before they can be applied for NCSs [5].

In recent years, there are many results for the stability of linear network control systems [6]-[9]. However, there is not much work reported in nonlinear networked control system analysis. Lyapunov functional, Lyapunov-Krasovski functional and Lyapunov-Razumikhin functional based methods are most widely used to study the stability of linear 
and nonlinear networked control systems where the problem is usually formulated as Linear Matrix Inequalities (LMIs). In most of the published work in the literature, the aim is to find the maximum allowable delay bound for a given nonlinearity bound and to increase the conservativene ss of the maximum allowable delay bound results but on the expense of increasing the complexity. In[10] Razumikh in and Lyapunov Theorem are used to derive a sufficient stability condition for the stability of a class of a nonlinear networked control system. The system under study is the linearized system with a bounded nonlinear function. A discrete-time approach for stabilizing a class of a nonlinear system is presented in[11] where the quadratic Lyapunov functional is used to derive a discrete linear controller for the affine nonlinear plant. In[12] a multi-input-multi-output continuous system is studied where the effects of the network induced time delay is modeled as an error state-vector which is regarded as a vanishing perturbation. The use of switching Lyapunov functional to derive stability conditions for a networked control system with bounded nonlinear uncertainty has been studied in[13]. $\operatorname{In}[14][15]$ the sampled-data approach is used for a networked control system with nonlinearity and the stability criteria is formulated as LMIs. Their method can be used to calculate the maximum nonlinearity bound for a given time delay and controller, but their results are conservative. The maximum nonlinear bound is calculated by solving a constrained optimization problem. The Lyapunov -Krasovski functional is used in[16] to derive LMI to study the stability and for designing a stabilizing controller for a networked control system with time-delay, drop-outs and bounded time-varying nonlinearity. The fuzzy-logic approach has been addressed in many papers[17]. The Authors in[17] modeled a class of nonlinear networked control system using Takagi-Sugeno model. They use the approximate model of the discrete nonlinear system to represent the actual system model. In[18] the authors provided new results for stability analysis and stabilization of linear systems with norm bounded nonlinear perturbation. Although the results of the maximum nonlinearity bound are less conservative, the method is limited to free delay systems.

Most of the previously developed approaches require excessive load of computations, and also for higher-order systems; the load of computations will increase dramatically. In practice, engineers may find it difficult to apply those available methods in control system design because of the complexity of the methods and the lack of a guideline in linking between the design parameters and the system performance. Furthermore, the design procedures highly depend on the post-design simulation to determine the design parameters. So there is a demand for a simple design approach with clear guidance for practical applications. The time delay in real-time networks depends strongly on the network protocol and by scheduling the network the time delay can be made smaller and bounded. In this paper, a new simple method is proposed for estimating the
Maximum Allowable Delay Bound (MADB) in NCS with bounded nonlinearity. The method depends on using the finite difference approximation of the delay term and the problem is formulated as LMI, which can be easily solved. Moreover, a simple analytical formula relating the MADB with the maximum nonlinearity bound is proposed.

The paper starts from the description of the proposed method for estimating the maximum time delay for NCS with norm bounded nonlinearity. A few examples are illustrated, and the results are compared with those proposed in the previously published literature.

\section{Mathematical Analysis}

A nonlinear system is given by:

$$
\dot{\mathbf{x}}(t)=\mathbf{A}_{p} \mathbf{x}(t)+\mathbf{B}_{p} \mathbf{u}(t)+h(t, \mathbf{x}(t))
$$

where $\mathbf{x}(t) \in \mathfrak{R}^{n}$ is the system state vector and $\mathbf{u}(t) \in \mathfrak{R}^{m}$ is the system control input. $\mathbf{A}_{p} \in \mathfrak{R}^{n \times n}$ and $\mathbf{B}_{p}$ $\in \mathfrak{R}^{n \times m}$ are matrices with appropriate sizes. $h(t, \mathbf{x}(t))$ is the nonlinearity.

The nonlinearity is assumed to be piecewise-continuous function of both $t$ and $\mathbf{x} . \quad h(t, \mathbf{x}(t))$ is uncertain and satis fies the quadratic inequality[14][15];

$$
h^{T}(t, \mathbf{x}(t)) h(t, \mathbf{x}(t)) \leq \alpha^{2} \mathbf{x}^{T}(t) \mathbf{H}^{T} \mathbf{H x}(t)
$$

where $\alpha>0$ is the nonlinearity bounding parameter and $\mathbf{H}$ is a constant matrix. For any given $\mathbf{H}$;

$$
\begin{gathered}
\mathbf{H}_{\alpha}=\left\{h: \mathbf{R}^{n+1} \rightarrow \mathbf{R}^{n} \mid h^{T}(t, \mathbf{x}(t)) h(t, \mathbf{x}(t))\right. \\
\left.\leq \alpha^{2} \mathbf{x}^{T}(t) \mathbf{H}^{T} \mathbf{H x}(t) \text { for all }(t, x) \in R_{+} \times \mathbf{R}^{n}\right\}
\end{gathered}
$$

The constraint (2) can be interpreted as [18];

$$
\|h(t, \mathbf{x}(t))\| \leq \alpha\|\mathbf{H} \mathbf{x}(t)\|
$$

Stabilizing the system with a linear controller which is given by;

$$
\mathbf{u}(t)=\mathbf{K x}(t-\tau)
$$

A typical networked control system model is shown in Figure 2. The time delay may be constant, variable or even random. In NCSs, the time delay is composed of the time delay from sensors to controllers, time delay in the controller and controllers to actuators time delay, which is given by:

$$
\tau=\tau_{s c}+\tau_{c}+\tau_{c a}
$$

where $\tau_{s c}$ is the time delay between the sensor and the controller $\tau_{c}$ is the time delay in the controller, $\tau_{c a}$ the time delay from the controller to the actuator. For a general formulation the packet dropouts can be incorporated in (5):

$$
\tau=\tau_{s c}+\tau_{c}+\tau_{c a}+d h
$$

where $d$ is the number of dropouts and $h$ the sampling period. And by (6) the data dropouts can be considered as a special case of the time delay[9].

It is supposed that the following hypotheses hold.

Hypothesis 1 (H.1):

- Sensors are clock driven. 
- The controllers and the actuators are event driven.

- The data are transmitted as a single packet.

- The old packets are discarded.

- All the states are available for measurements and hence for transmission.

- The time delay $\tau$ is small enough for the finite difference approximation to be hold.

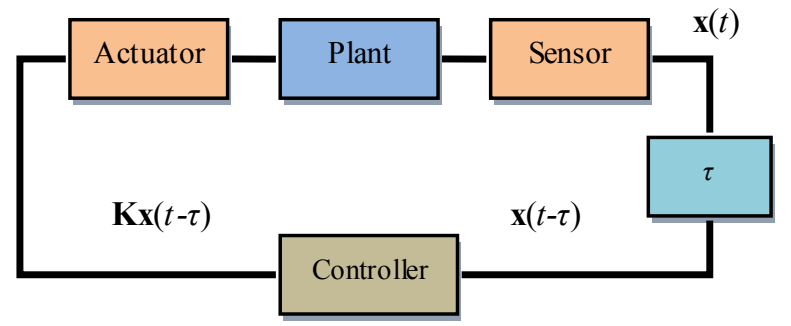

Figure 2. An NCS with time delay between the sensor and the controller.

Before we proceed to the analysis we will use the following Lemma:

Lemma 1:(Schur Complement):[4]

For a given symmetric matrix

$$
\boldsymbol{\Omega}=\left[\begin{array}{ll}
\boldsymbol{\Omega}_{11} & \boldsymbol{\Omega}_{12} \\
\boldsymbol{\Omega}_{12}{ }^{T} & \boldsymbol{\Omega}_{22}
\end{array}\right]
$$

where $\boldsymbol{\Omega}_{11}, \boldsymbol{\Omega}_{12}$ and $\boldsymbol{\Omega}_{22}$ are block matrices, and $\boldsymbol{\Omega}_{11}$ is a square matrix. The following three conditions are equal in value:

$$
\begin{gathered}
\boldsymbol{\Omega}<0 \\
\boldsymbol{\Omega}_{22}<0, \quad \boldsymbol{\Omega}_{11}-\boldsymbol{\Omega}_{12} \boldsymbol{\Omega}_{22}{ }^{-1} \boldsymbol{\Omega}_{12}{ }^{T}<0 \\
\boldsymbol{\Omega}_{11}<0, \quad \boldsymbol{\Omega}_{22}-\boldsymbol{\Omega}_{12}{ }^{T} \boldsymbol{\Omega}_{11}{ }^{-1} \boldsymbol{\Omega}_{12}<0
\end{gathered}
$$

Applying (4) into (1);

$$
\begin{aligned}
\dot{\mathbf{x}}(t) & =\mathbf{A}_{p} \mathbf{x}(t)+\mathbf{B}_{p} \mathbf{K} \mathbf{x}(t-\tau)+h(t, \mathbf{x}(t)) \\
\dot{\mathbf{x}}(t) & =\left(\mathbf{A}_{p}+\mathbf{B}_{p} \mathbf{K}\right) \mathbf{x}(t) \\
& +\mathbf{B}_{p} \mathbf{K}(\mathbf{x}(t-\tau)-\mathbf{x}(t))+h(t, \mathbf{x}(t))
\end{aligned}
$$

If H. 1 holds, then the time delay term can be approximated using the finite difference approximation by Tyler Series expansion. The exp ression for $\mathbf{x}(t-\tau)$ can be obtained by Taylor Expansion as:

$$
\mathbf{x}(t-\tau)=\sum_{n=0}^{\infty}(-1)^{n} \frac{\tau^{n}}{n !} \mathbf{x}(t)
$$

Where $\mathbf{x}^{(n)}(t)$ is the $\mathrm{n}^{\text {th }}$ order derivative. The second order approximation of the delay term is given by;

$$
\begin{gathered}
\mathbf{x}(t-\tau)=\mathbf{x}(t)-\tau \dot{\mathbf{x}}(t)+\left(\tau^{2} / 2\right) \ddot{\mathbf{x}}(t)+\mathbf{R}_{3}(\mathbf{x}, \tau) \\
\mathbf{x}(t-\tau) \approx \mathbf{x}(t)-\tau \dot{\mathbf{x}}(t)+\left(\tau^{2} / 2\right) \ddot{\mathbf{x}}(t)
\end{gathered}
$$

From (9) it can be seen that $\mathbf{R}_{3}(\mathbf{x}, \tau)$ depends on the time delay, $\tau$, and the higher-order derivatives of $\mathbf{x}(t)$ which can be neglected if the time delay and the norm of $\mathbf{R}_{3}(\mathbf{x}, \tau)$ are small. For small time delay and slowly time varying nonlinear perturbation the second derivative can be approximated as:

$$
\ddot{\mathbf{x}}(t) \approx\left(\mathbf{A}_{p}+\mathbf{B}_{p} \mathbf{K}\right) \dot{\mathbf{x}}(t)
$$

Substituting (9) and (10) into (7);

$$
\begin{aligned}
\dot{\mathbf{x}}(t) \cong & \left(\mathbf{A}_{p}+\mathbf{B}_{p} \mathbf{K}\right) \mathbf{x}(t) \\
& +\mathbf{B}_{p} \mathbf{K}\left(-\tau \dot{\mathbf{x}}(t)+\left(\tau^{2} / 2\right)\left(\mathbf{A}_{p}+\mathbf{B}_{p} \mathbf{K}\right) \dot{\mathbf{x}}(t)\right)+h(t, \mathbf{x}(t))
\end{aligned}
$$

Equation (11) can be written as:

$$
\dot{\mathbf{x}}(t) \cong \mathbf{N}(\tau)\left(\mathbf{A}_{p}+\mathbf{B}_{p} \mathbf{K}\right) \mathbf{x}(t)+g(t, \mathbf{x}(t))
$$

where;

$$
\begin{aligned}
g(t, \mathbf{x}(t)) & =\mathbf{N}(\tau) h(t, \mathbf{x}(t))=[\mathbf{I}+\mathbf{M}(\tau)]^{-1} h(t, \mathbf{x}(t)) \\
& =\left[\mathbf{I}+\tau \mathbf{B}_{p} \mathbf{K}\left(\mathbf{I}-0.5 \tau\left(\mathbf{A}_{p}+\mathbf{B}_{p} \mathbf{K}\right)\right)\right]^{-1} h(t, \mathbf{x}(t))
\end{aligned}
$$

According to (2) with the time delay the quadratic inequality can be written as;

$$
\begin{aligned}
& g^{T}(t, \mathbf{x}(t)) g(t, \mathbf{x}(t)) \\
& \leq \alpha^{2} \mathbf{x}^{T}(t) \mathbf{H}^{T} \mathbf{N}(\tau)^{T} \mathbf{N}(\tau) \mathbf{H} \mathbf{x}(t)
\end{aligned}
$$

which can be interpreted as;

$$
\|g(t, \mathbf{x}(t))\| \leq \alpha\|\mathbf{N}(\tau) \mathbf{H} \mathbf{x}(t)\| \leq \alpha\|\mathbf{N}(\tau)\| \mathbf{H}\|\mid\| \mathbf{x}(t) \|
$$

The constraint can be written as;

$$
\mathbf{z}(t)^{T}\left[\begin{array}{cc}
-\alpha^{2} \mathbf{H}^{T} \mathbf{N}(\tau)^{T} \mathbf{N}(\tau) \mathbf{H} & 0 \\
0 & \mathbf{I}
\end{array}\right] \mathbf{z}(t) \leq 0
$$

where; $\mathbf{z}(t)=\left[\begin{array}{c}\mathbf{x}(t) \\ g(t, x(t))\end{array}\right]$

Choosing the quadratic Lyapunov functional candidate and taking its derivative;

$$
\begin{gathered}
\dot{\mathbf{V}}(x)=\mathbf{x}^{\mathrm{T}}(t) \mathbf{P} \dot{\mathbf{x}}(t)+\dot{\mathbf{x}}^{\mathrm{T}}(t) \mathbf{P} \mathbf{x}(t) \\
=\mathbf{x}^{\mathrm{T}} \mathbf{P}\left(\mathbf{N}(\tau)\left(\mathbf{A}_{p}+\mathbf{B}_{p} \mathbf{K}\right) \mathbf{x}(t)+g(t, \mathbf{x}(t))\right) \\
+\left(\mathbf{x}(t)^{T}\left(\mathbf{A}_{p}+\mathbf{B}_{p} \mathbf{K}\right)^{T} \mathbf{N}(\tau)^{T}+g^{T}(t, \mathbf{x}(t))\right) \mathbf{P} \mathbf{x} \\
\dot{\mathbf{V}}(x)=\mathbf{x}(t)^{T}\left(\mathbf{P N}(\tau)\left(\mathbf{A}_{p}+\mathbf{B}_{p} \mathbf{K}\right)\right. \\
\left.+\left(\mathbf{A}_{p}+\mathbf{B}_{p} \mathbf{K}\right)^{T} \mathbf{N}(\tau)^{T} \mathbf{P}\right) \mathbf{x}(t) \\
+g^{T}(t, \mathbf{x}(t)) \mathbf{P} \mathbf{x}(t)+\mathbf{x}(t)^{T} \mathbf{P} g(t, \mathbf{x}(t))
\end{gathered}
$$

This can be written as;

$$
\begin{aligned}
\dot{\mathbf{x}}(t) \cong & {\left[\mathbf{I}+\tau \mathbf{B}_{p} \mathbf{K}\left(\mathbf{I}-0.5 \tau\left(\mathbf{A}_{p}+\mathbf{B}_{p} \mathbf{K}\right)\right)\right]^{-1}\left(\mathbf{A}_{p}+\mathbf{B}_{p} \mathbf{K}\right) \mathbf{x}(t) } \\
& +\left[\mathbf{I}+\tau \mathbf{B}_{p} \mathbf{K}\left(\mathbf{I}-0.5 \tau\left(\mathbf{A}_{p}+\mathbf{B}_{p} \mathbf{K}\right)\right)\right]^{-1} h(t, \mathbf{x}(t)) \\
& \mathbf{z}(t)^{T}\left[\begin{array}{cc}
\mathbf{A}^{T} \mathbf{N}(\tau)^{T} \mathbf{P}+\mathbf{P N}(\tau) \mathbf{A} & \mathbf{P} \\
\mathbf{P} & \mathbf{0}
\end{array}\right] \mathbf{z}(t)<0
\end{aligned}
$$

Where; $\mathbf{A}=\left(\mathbf{A}_{p}+\mathbf{B}_{p} \mathbf{K}\right)$

Following the approach in[18] by combining (15) and (17) we get;

$$
\left[\begin{array}{cc}
\mathbf{A}^{T} \mathbf{N}(\tau)^{T} \mathbf{P}+\mathbf{P N}(\tau) \mathbf{A}+\varepsilon \alpha^{2} \mathbf{H}^{T} \mathbf{N}(\tau)^{T} \mathbf{N}(\tau) \mathbf{H} & \mathbf{P} \\
\mathbf{P} & -\varepsilon \mathbf{I}
\end{array}\right]<0
$$

Letting $\mathbf{Y}=\varepsilon \cdot \mathbf{P}^{-1}, \varepsilon>0$, and using Lemma 1 we finally get;

$$
\left[\begin{array}{ccc}
\mathbf{N}(\tau) \mathbf{A Y}+\mathbf{Y} \mathbf{A}^{T} \mathbf{N}(\tau)^{T} & \mathbf{I} & \mathbf{Y} \mathbf{H}^{T} \mathbf{N}(\tau)^{T} \\
\mathbf{I} & -\mathbf{I} & \mathbf{0} \\
\mathbf{N}(\tau) \mathbf{H Y} & \mathbf{0} & -\boldsymbol{\gamma} \mathbf{I}
\end{array}\right]<0
$$


where $\gamma=1 / \alpha^{2}$

\section{The orem 1}

System (1) and the controller (4) with a given time delay is robustly stable with degree $\alpha$ if the following is feasible

Minimize $\gamma$

Subject to $\mathrm{Y}>0$ and (18)

The optimization problem in Theorem 1 is quasi-convex optimization in $\gamma$ which can be solved easily using the Matlab LMI Toolbox. For systems with small time delays where the first derivative approximation can be used, the matrix $\mathbf{M}(\tau)$ can be approximated as $\mathbf{M}(\tau) \approx \tau \mathbf{B K}$, which can lead to less conservative results.

\section{Cor ollary 1}

Let H. 1 holds, then the nonlinear system (1) with the controller (4) is robustly stable with degree $\alpha$ if

\section{Proof}

$$
\alpha<\frac{\lambda_{\min }(\mathbf{Q})}{2\|\mathbf{P}\|_{2} \cdot\|\mathbf{H}\|_{2} \cdot\|\mathbf{N}(\tau)\|}
$$

Choosing a Lyapunov functional candidate as:

$$
\mathbf{V}(x)=\mathbf{x}^{\mathrm{T}} \mathbf{P} \mathbf{x}>0, \quad \forall \mathbf{x} \neq \mathbf{0}
$$

The objective for the next step is to find the range of $\tau$ that will ensure $\dot{\mathrm{V}}(x)<0$ for $\forall \mathbf{x} \neq \mathbf{0}$ [19][20]. Taking the derivative of (19) along with the system trajectory (12),

$$
\begin{gathered}
\dot{\mathbf{V}}(x)=\mathbf{x}^{\mathrm{T}}(t) \mathbf{P} \dot{\mathbf{x}}(t)+\dot{\mathbf{x}}^{\mathrm{T}}(t) \mathbf{P} \mathbf{x}(t) \\
=\mathbf{x}^{\mathrm{T}} \mathbf{P}\left(\mathbf{N}(\tau)\left(\mathbf{A}_{p}+\mathbf{B}_{p} \mathbf{K}\right) \mathbf{x}(t)+\mathbf{N}(\tau) h(t, \mathbf{x}(t))\right) \\
+\left(\mathbf{x}(t)^{T}\left(\mathbf{A}_{p}+\mathbf{B}_{p} \mathbf{K}\right)^{T} \mathbf{N}(\tau)^{T}+h^{T}(t, \mathbf{x}(t)) \mathbf{N}(\tau)^{T}\right) \mathbf{P} \mathbf{x} \\
=\mathbf{x}^{\mathrm{T}}(t) \mathbf{P N}(\tau)\left(\mathbf{A}_{p}+\mathbf{B}_{p} \mathbf{K}\right) \mathbf{x}(t) \\
+\mathbf{x}(t)^{T}\left(\mathbf{A}_{p}+\mathbf{B}_{p} \mathbf{K}\right)^{T} \mathbf{N}(\tau)^{T} \mathbf{P} \mathbf{x}(t)+2 \mathbf{x}^{\mathrm{T}}(t) \mathbf{P N}(\tau) h(t, \mathbf{x}(t)) \\
=\mathbf{x}^{\mathrm{T}}(t)\left(\mathbf{P N}(\tau)\left(\mathbf{A}_{p}+\mathbf{B}_{p} \mathbf{K}\right)+\left(\mathbf{A}_{p}+\mathbf{B}_{p} \mathbf{K}\right)^{T} \mathbf{N}(\tau)^{T} \mathbf{P}\right) \mathbf{x}(t) \\
+2 \mathbf{x}^{\mathrm{T}}(t) \mathbf{P N}(\tau) h(t, \mathbf{x}(t))
\end{gathered}
$$

If there exists $\mathbf{P}=\mathbf{P}^{T}>0$ and $\mathbf{Q}=\mathbf{Q}^{T}>0$, satisfying:

$$
\mathbf{P N}(\tau)\left(\mathbf{A}_{p}+\mathbf{B}_{p} \mathbf{K}\right)+\left(\mathbf{A}_{p}+\mathbf{B}_{p} \mathbf{K}\right)^{T} \mathbf{N}(\tau)^{T} \mathbf{P}=-\mathbf{Q}
$$

Substituting (21) into (20) we get:

$$
\dot{\mathbf{V}}(\mathbf{x}(t))=-\mathbf{x}^{\mathrm{T}}(t) \mathbf{Q} \mathbf{x}(t)+2 \mathbf{x}^{\mathrm{T}}(t) \mathbf{P N}(\tau) h(t, \mathbf{x}(t))
$$

For any $\alpha>0$, there exist $r>0$ such that

$$
\| h\left(t, \mathbf{x}(t)\left\|_{2}<\alpha\right\| \mathbf{H}\|\cdot\| \mathbf{x}(t)\left\|_{2}, \quad \forall\right\| \mathbf{x}(t) \|_{2}<r\right.
$$

Then;

$$
\begin{aligned}
\| \mathbf{N}(\tau) h(t, \mathbf{x}(t) \| & <\|\mathbf{N}(\tau)\| \cdot \| h(t, \mathbf{x}(t) \| \\
& <\alpha\|\mathbf{N}(\tau)\| \cdot\|\mathbf{H}\| \cdot\|\mathbf{x}(t)\|_{2}
\end{aligned}
$$

Also we have[21];

$$
\mathbf{x}^{T}(t) \mathbf{Q} \mathbf{x}(t) \geq \lambda_{\min }(\mathbf{Q})\|\mathbf{x}(t)\|_{2}^{2}
$$

Using (23) and (24) into (22) we finally have;

$$
\dot{\mathbf{V}}(\mathbf{x}(t))<-\left[\lambda_{\text {min }}(\mathbf{Q})-2 \alpha\|\mathbf{P}\|_{2}\|\mathbf{H}\|_{2}\|\mathbf{N}(\tau)\|\right] \mid \mathbf{x}(t) \|_{2}^{2}
$$

From(25) it can be found that if

$$
\alpha<\frac{\lambda_{\min }(\mathbf{Q})}{2\|\mathbf{P}\|_{2}\|\mathbf{H}\|_{2} \cdot\|\mathbf{N}(\tau)\|}
$$

then $\dot{\mathrm{V}}(x)<0$, the system will be robustly stable with degree $\alpha$. We can see from Corollary 1 that the MADB decreases with increasing $\alpha$. Setting $\alpha \approx 0$ and neglecting the second order term then Corollary 1 reduces to Corollary 1 in[22] as follows;

$$
\begin{gathered}
\tau<\frac{1}{\left\|\mathbf{B}_{p} \mathbf{K}\right\|}\left[1-\frac{2 \alpha\|\mathbf{P}\|_{2} \cdot\|\mathbf{H}\|_{2}}{\lambda_{\min }(\mathbf{Q})}\right] \\
\tau<\frac{1}{\left\|\mathbf{B}_{p} \mathbf{K}\right\|}\left[1-0 \frac{2\|\mathbf{P}\|_{2} \cdot\|\mathbf{H}\|_{2}}{\lambda_{\min }(\mathbf{Q})}\right] \Rightarrow \tau<\frac{1}{\left\|\mathbf{B}_{p} \mathbf{K}\right\|}
\end{gathered}
$$

Increasing $\alpha$ means moving away from the equilibrium point. We noticed as we move away from the equilibrium point the MADB decreases. The boundary of the domain of attraction is when:

$$
\alpha \approx \lambda_{\min }(\mathbf{Q}) /\left[2\|\mathbf{P}\|_{2} \cdot\|\mathbf{H}\|_{2}\right] \Rightarrow \tau<(1-1) /\left\|\mathbf{B}_{p} \mathbf{K}\right\| \Rightarrow 0<\tau<0
$$

which means the MADB on the boundary is approximately zero.

In NCS with nonlinearity it is important to find or estimate the domain of attraction. The domain of attraction is defined as the region where the limit of every trajectory of the nonlinear system orig inating in $R_{A}$ is the equilibrium point. $R_{A}$ is shown in Figure 3. It is assumed that the origin is asymptotically stable.

In[21] the domain of attraction of the equilibrium point (the origin) is defined as:

$$
R_{A}=\left\{x \in R^{n} \mid \phi(t ; x) \rightarrow 0 \text { as } t \rightarrow \infty\right\}
$$

where $\phi(t ; x)$ is the initial state at $t=0$. It is difficult to find the do ma in of attraction but we can estimate a region $\Omega_{\mathrm{c}}$, that is $\Omega_{\mathrm{c}} \subset R_{A}$, using Lyapunov's method. The estimate of the domain of attraction $\Omega_{\mathrm{c}}$ in [21] is defined as:

$$
\begin{array}{r}
\Omega_{c}=\left\{x \in R^{n} \mid V(x) \leq c\right\} \\
c<\min _{\|x\|_{2}=r} \mathbf{V}(x)
\end{array}
$$

where $c$ is a positive constant. Since;

$$
\mathbf{x}^{T} \mathbf{P}(\tau) \mathbf{x} \geq \lambda_{\min }(\mathbf{P}(\tau))\|x\|_{2}^{2}
$$

For $\mathbf{V}(x)$ to be positive $\mathrm{c}$ can be chosen as:

$$
c \leq \lambda_{\min }(\mathbf{P}(\tau)) r^{2}
$$

From (28) we can draw some conclusions on the relation between the time delay and the domain of attraction because of the dependence of $\lambda_{\min }(\mathbf{P}(\tau))$ on the time delay through (21). A few references[13][14] reported that increasing the 
time de lay decreases the nonlinearity bound. In the following section, a number of examples are picked-up from literature for comparison and discussion.

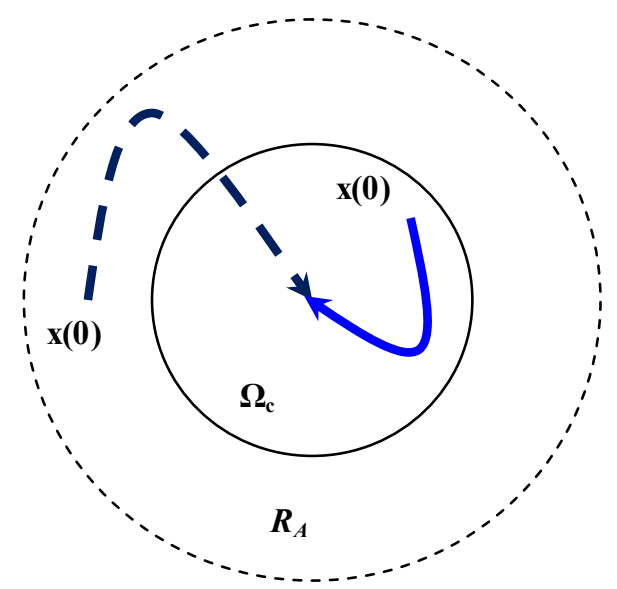

Figure 3. The region of attraction.

\section{Stability Analysis Case Studies}

In general, two approaches are applied to controller design for NCSs. The first design approach is to estimate the maximu $\mathrm{m}$ allowable delay bound for the system and then the network is scheduled to limit the time delay to be less than the MADB. The second approach is to design the controller while taking the time delay and data dropouts into account. In this paper, the first approach has been adopted. In this section, a number of examp les are studied to demonstrate the approach proposed and compare it with the previously published cases. In particular, the results derived using the method proposed in this paper has been compared with the results using the LMI method given in[14][15].

Example 1

The first example has been studied in[14][15] with the sampled-data approach, the system is given by

$$
\dot{\mathbf{x}}(t)=\left[\begin{array}{cc}
1 & 1 \\
0 & 0.99
\end{array}\right] \mathbf{x}(t)+\left[\begin{array}{c}
0 \\
10
\end{array}\right] \mathbf{u}(t)+h(t, \mathbf{x}(t))
$$

with $\mathbf{H}=\left[\begin{array}{ll}1 & 0 \\ 0 & 0\end{array}\right]$

The controller is chosen in[14][15] to be $\mathbf{K}=\left[\begin{array}{ll}-0.2999 & -0.2989\end{array}\right]$. With $\alpha \approx 0$ using Theorem 1 the MADB is $0.292 \mathrm{~s}$. For a time delay, $\tau=0.2509 \mathrm{~s}$, and using Theorem 1 we have:

$$
\alpha_{\max }=0.1533 \text { with } \mathbf{Y}=\left[\begin{array}{cc}
8.191 & -48.094 \\
-48.094 & 311.1583
\end{array}\right]
$$

Using Corollary 1:

$$
\begin{gathered}
\mathbf{P}=\left[\begin{array}{cc}
0.7213 & -1.2213 \\
-1.2213 & 2.4487
\end{array}\right], \mathrm{Q}=\mathrm{I}\|\mathbf{P}\|_{2}=3.0809 \\
\lambda_{\text {max }}(\mathbf{P})=3.0809, \alpha_{\text {max }}=0.0184
\end{gathered}
$$

The maximum nonlinearity bound given in[14][15] is 0.0013. $\operatorname{In}[24] \alpha_{\max }=0.1636$ with $0.2509 \mathrm{~s}$ time delay. However Corollary 1 and Theorem 1 still give conservative results the method is very easy compared with the method in[14],[15] and[24]. It is clear that the results of Theorem 1 are less conservative than the results of Corollary 1.The trajectory of the system is shown in Figure 4. For comparison the nonlinear function and the initial conditions for the simulation are given by;

$$
h(t, \mathbf{x}(t))=\left[\begin{array}{c}
\alpha x_{1} \sin \left(x_{1}\right) \\
0
\end{array}\right] \quad \mathbf{x}(0)=\left[\begin{array}{ll}
-15 & 10
\end{array}\right]^{T}
$$

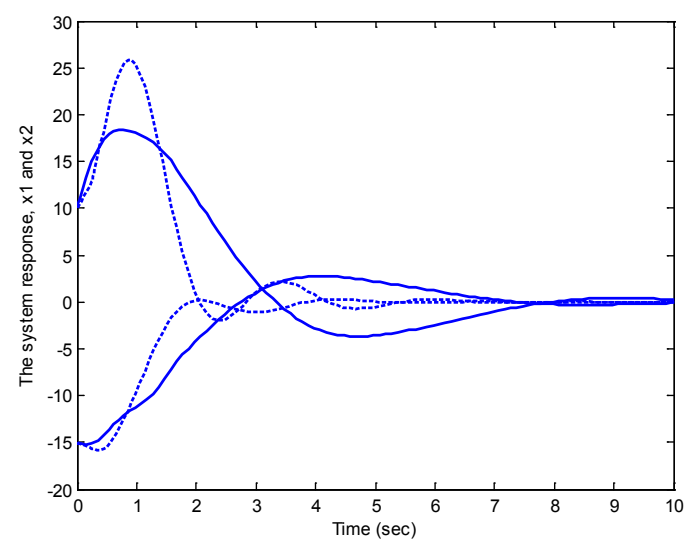

Figure 4. The system response with zero time delay (solid-line) and 0.2509 s time delay (dashed-line)

$\operatorname{In}[14],[15]$ with $0.22 \mathrm{~s}$ time delay and $\mathbf{K}=\left[\begin{array}{ll}-0.359 & -0.317\end{array}\right]$, the maximum nonlinear bound is $\alpha_{\max }=0.1365$, using Corollary $1 \alpha_{\max }=0.0256$ while using Theorem $1 \alpha_{\max }=0.2555$. Here Theorem 1 gives less conservative results than the published ones. The MADB as a function of the nonlinearity is given in Figure 5. It can be easily seen that as the nonlinearity increases the MADB decreases.

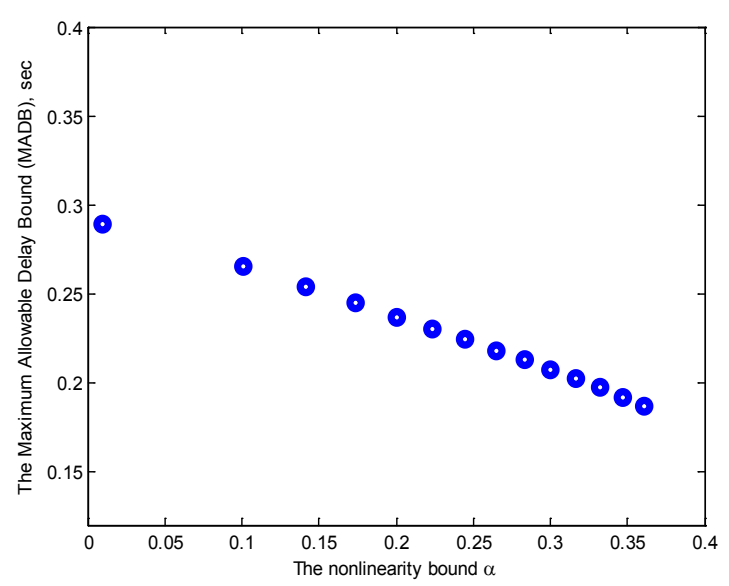

Figure 5. The MADB as a function of the nonlinearity bound using Theorem 1

\section{Example 2}

This example has been studied in[17] with the sampled-data approach, the system is given by 


$$
\dot{\mathbf{x}}(t)=\left[\begin{array}{ccc}
0 & 1 & 0 \\
0 & -0.5 & 1 \\
3 & 3 & -2.5
\end{array}\right] \mathbf{x}(t)+\left[\begin{array}{c}
0 \\
1 \\
0.6
\end{array}\right] \mathbf{u}(t)+\left[\begin{array}{c}
0 \\
0 \\
x_{1}(t) \sin \left(x_{1}(t)\right)
\end{array}\right]
$$

The controller in[17] is designed to be $\mathbf{K}=\left[\begin{array}{lll}-9.0255 & -9.3741 & -5.1724\end{array}\right]$. Setting $\alpha \approx 0$ and using theorem 1 the MADB is $0.0601 \mathrm{~s}$. The maximum nonlinearity bound for the delay free system is 4.3. The MADB as a function of the nonlinearity is shown in Figure 6. The system response with $0.03 \mathrm{~s}$ and 3 nonlinearity is shown in Figure 7.

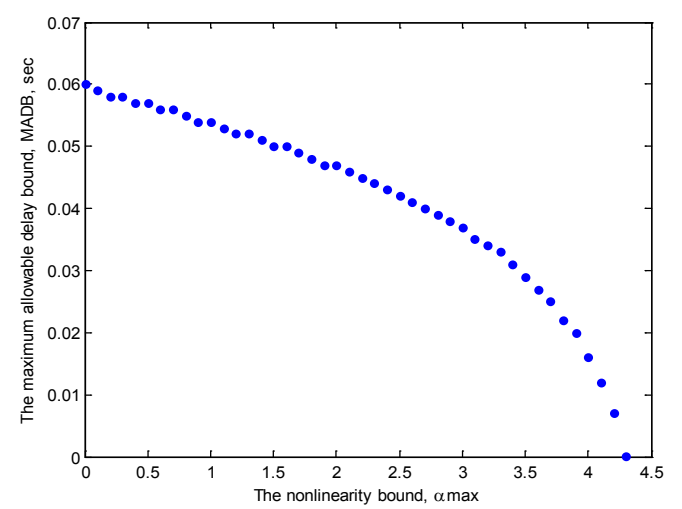

Figure 6. The MADB as a function of the maximum nonlinearity bound using Theorem 1

From Figure 5 and Figure 6 it is clear that increasing the nonlinearity bound decreases the MADB. The same relation has been noticed in[14][15]. We have carried out many simulation and we found that increasing the nonlinearity reduces the MADB. Although the method is still conservative, but it can be easily applied.

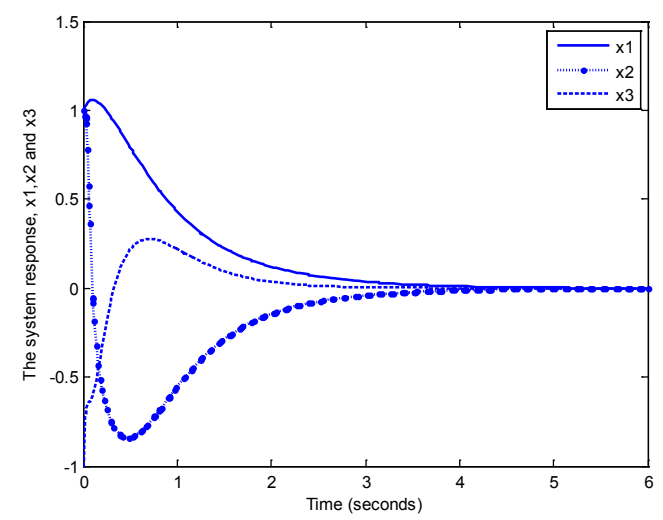

Figure 7. The system response with $0.03 \mathrm{~s}$ time delay and $\alpha=3$

Example 3:

The last example is the estimation of the region of attraction using the proposed method. A nonlinear system is given by:

$$
\dot{x}(t)=x^{2}(t)+u(t)
$$

$\operatorname{In}[10]$ the system is stabilized with a linear controller as:

$$
u(t)=-\frac{1}{2} x(t-\tau)
$$

In[10] the authors use Razu mikh in and Lyapunov theorem and the MADB reported in[10] is $2 \mathrm{~s}$. Using Theorem 1 with $\mathbf{H}=1, \mathbf{K}=-1 / 2$ and $\mathbf{M}(\tau) \approx \tau \mathbf{B K}$, the MADB is $2 \mathrm{~s}$. Choosing $\mathbf{K}=-2$; from (21) we have;

$$
\mathbf{P N}(\tau)[\mathbf{A}+\mathbf{B K}]+[\mathbf{A}+\mathbf{B K}]^{T} \mathbf{N}(\tau)^{-T} \mathbf{P}=-\mathbf{Q}
$$

Choosing $\mathbf{Q}=1$;

$$
\mathbf{P}=\frac{1}{4}(1-2 \tau(1+\tau))
$$

For $\mathbf{P}$ to be positive $\tau<0.366$, so the MADB is $0.366 \mathrm{~s}$. Using Theorem 1 with $\mathbf{H}=1$, the MADB is $0.366 \mathrm{~s}$.

Using the finite difference approximation for the delay term, the time delay nonlinear system is given by;

$$
\dot{x}(t)=(1-2 \tau(1+\tau))^{-1}\left(x^{2}(t)-2 x(t)\right)
$$

The system is stable if $\tau<0.366$. Choosing Lyapunov functional candidate as;

$$
\begin{gathered}
\mathbf{V}(x)=\mathbf{x}^{\mathrm{T}} \mathbf{P} \mathbf{x}>0, \quad \forall \mathbf{x} \neq \mathbf{0} \\
\dot{\mathbf{V}}(x)=\dot{\mathbf{x}}^{\mathrm{T}} \mathbf{P} \mathbf{x}+\mathbf{x}^{\mathrm{T}} \mathbf{P} \dot{\mathbf{x}} \\
\dot{V}(x)=2(1-2 \tau(1+\tau))^{-1} x(t) P(x(t)-2) x(t) \\
=2(1-2 \tau(1+\tau))^{-1} x^{2}(t) P(x(t)-2) \\
\mathbf{x}(\mathrm{t})<2, \quad \dot{V}(x)<0
\end{gathered}
$$

The equilibrium points of the system are: $x=0$ and $x=2$, we will study the domain of the attraction at the orig in; for the system to be stable we must have;

$$
x(t)<2 \text { that implies } x^{2}(t)<4
$$

The doma in of attraction is estimated through using (28); $c \leq \lambda_{\min }(\mathbf{P}) r^{2}$ where $r=2$ and $\mathbf{P}=\frac{1}{4}(1-2 \tau(1+\tau))$,

$$
c \leq \frac{1}{4}(1-2 \tau(1+\tau)) \cdot 2^{2} \text { that implies } \quad c \leq(1-2 \tau(1+\tau))
$$

We can see that increasing the time delay decreases the domain of attraction and when the time delay approaches the MADB then the domain of attraction becomes very small.

The system response with different time delays is shown in Figure 8. From Figure 8, the system is still stable even with $0.75 \mathrm{~s}$, which shows that the results of Theorem 1 are still conservative. Figure 9 and Figure 10 show the system response with $0.76 \mathrm{~s}$ time delay and 0.15 and 0.5 initial condition respectively.

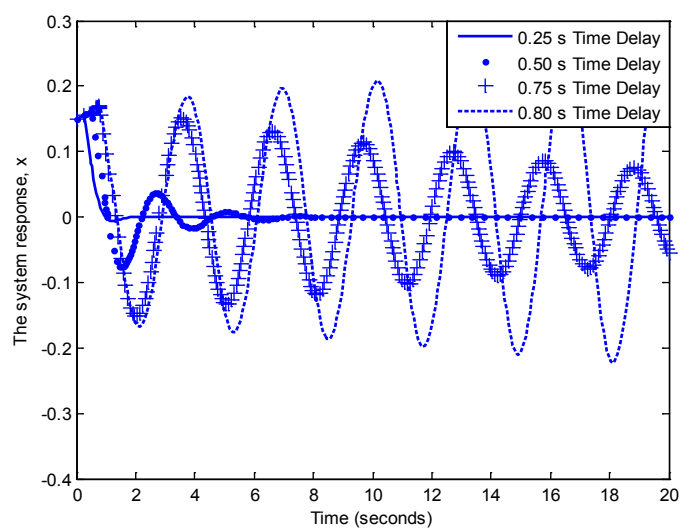

Figure 8. The system response with different time delays and 0.15 initial condition 


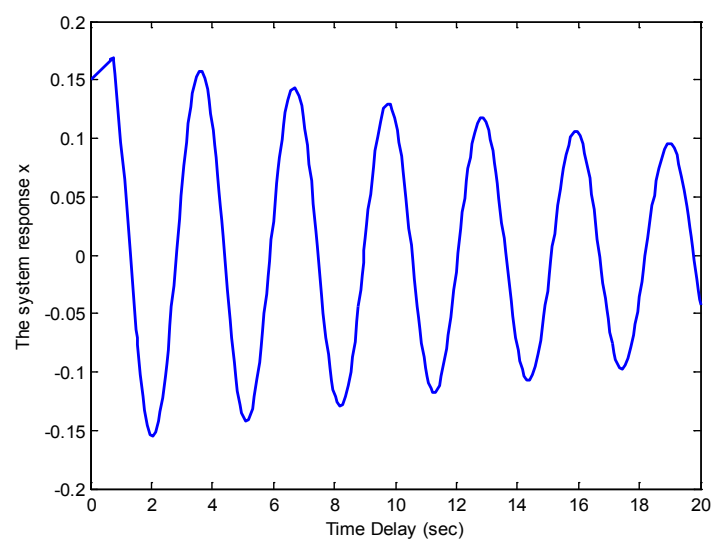

Figure 9. The system response with $0.76 \mathrm{~s} \mathrm{time} \mathrm{delay} \mathrm{and} 0.15$ initial condition

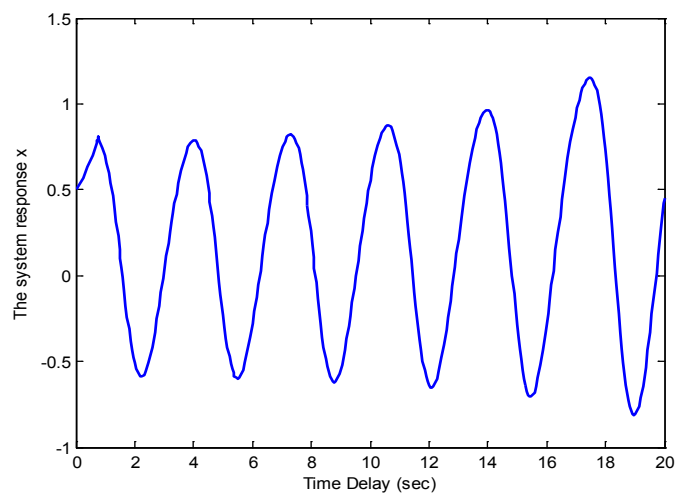

Figure 10. The system response with $0.76 \mathrm{~s} \mathrm{time} \mathrm{delay} \mathrm{and} 0.5$ initial condition

In Figure 9, the initial condition is 0.15 , and we can see that the system is stable while in Figure 10 the initial condition is 0.5 , and the system is unstable. Increasing the initial condition reduces the MADB, and this is the same conclusion obtained from Corollary 1 also in[10] the authors show that increasing the time delay reduces the domain of attraction.

\section{Conclusions}

The main contribution of the paper is to have derived a new method for estimating the maximum time delay in NCSs with norm bounded nonlinearity. The most attractive feature of the new method is that it is simple in structure and easy for applications, which can be clearly interpreted to design engineers in industrial sectors. The results obtained in this method are compared with those obtained through the methods introduced in other literatures. The method has demonstrated its merits in using less computation time due to its simple structure and giving less conservative results while showing good agreement with other methods. The method is used to estimate the MADB for a given nonlinearity bound which can be used as a guiding tool for the network scheduling. We found that increasing the nonlinearity bound reduces the MADB also increasing the time delay reduces the domain of the attraction for the NCS with bounded nonlinearity.

\section{REFERENCES}

[1] Xiefu Jiang, Qing-Long Han, Shirong Liu, and Anke Xue. "A New $\mathrm{H} \infty$ Stabilization Criterion for Networked Control Systems", IEEE Transactions On Automatic Control, Vol.53, No.4, 1025-1032. May 2008.

[2] Gregory C. Walsh and Hong Ye and Linda Bushnell. "Stability Analysis of Networked Control Systems", The Proceedings of the American Control Conference, San Diego, California, USA, Vol. 4, pp. 2876-2880, June 1999.

[3] Gregory C. Walsh, Hong Ye, and Linda G. Bushnell. "Stability analysis of networked control systems," IEEE Transactions on Control Sy stem Technology, Vol. 10, No. 3, 438-446, May 2002.

[4] Magdi. S. Mahmoud, "Robust Control and Filtering For Time Delay systems", New York: Marcel Dekker, 2000.

[5] Magdi S. Mahmoud*, Abdulla Ismail. "Role of Delays in Networked Control Systems", Proceedings of the 10th IEEE International Conference on Electronic Circuits and Systems, Volume 1, 40-43, 2003.

[6] Dong Yue, Qing-Long Han, and Chen Peng., "State Feedback Controller Design of Networked Control Systems", IEEE Transactions on Circuits and Systems, Vol. 51, No. 11, 640-644, November 2004.

[7] Bin Tang, Guo-Ping Liu, and Wei-Hua Gui. "Improvement of State Feedback Controller Design for Networked Control Systems", IEEE Transactions on Circuits and Systems, Vol. 55, No. 5, 464-468, May 2008.

[8] Sun Jian, Liu Guoping, Chen Jie "State Feedback Stabilization of Networked Control Systems", Proceedings of the 27th Chinese Control Conference, Kunming, Yunnan, China, 457-461, July 2008.

[9] Yuquan Zhang, Qiuhai Zhong, and Lei Wei, L. "Stability Analysis of Networked Control Systems with Communication Constraints", The Proceedings of Chines Control and Decision Conference, 335-339, 2008.

[10] Jun Yang and Xiangdong Wang, "Stability of a Class of Nonlinear Networked Control Systems", The Proceedings of the 5th World Congress on Intelligent Control and Automation, China, 1401-1405,2004.

[11] Dan Man, Georgi M. Dimirovski, Jovan D. Stefanovski, and Jun Zhao, "Exponential Stability Synthesis of Networked Nonlinear Control Systems in FMS", 414-419.

[12] Gregory. C. Walsh, Octavian Beldiman, and Linda G. Bushnell, "Asymptotic Behavior of Nonlinear Networked Control Systems", 1093-1097, IEEE Transactions on Automatic Control, Vol. 46, No. 7,, July 2007.

[13] Junyan Yu, Long Wang, Mei Yu, Jie Chen and Yingmin Jia, "Robust Controller Design for Networked Control Systems with Nonlinear Uncertainties", 2803-2808, In the Proceedings of the 2009 American Control Conference.

[14] M. Yu, L. Wang and T. Chu, "Sampled-data Stabilisation of Networked Control Systems with Nonlinearity", 609-614, 
IEE Proc Control Theory Applications, Vol. 152, No. 6, November 2005.

[15] M. Yu, L. Wang and T. Chu, "Robust Stabilization of Nonlinear Sampled-data Systems", 3421-3426, In the Proceeding of the 2005 American Control Conference, Portland, USA, 2005.

[16] Jian Sun and G. P. Liu, "Robust Stabilization of a Class of Nonlinear Networked Control Systems", 2035-2040, In the Proceedings of the 25th Chinese Control Conference, 2006

[17] Yang Wang, Bin Jiang, and Zehui Mao, "Fault-tolerant Control Design for a kind of Nonlinear Networked Control System with Communication Constraints", 896-901, In the Proceedings of the Chinese Control and Decision Conference, 2009.

[18] D. D. Siljak and D. M. Stipanovic, "Robust Stabilization of Nonlinear Systems: The LMI Approach", The Journal of Mathematical Problems in En gineering, 461-493, Vol. 6.

[19] D. P. Goodall, and J. Wang. "Stabilisation of a class of uncertain non-linear affine control systems subject to control constraints", International Journal of Robust and Nonlinear Control, Vol.10, 797-818, 2001.
[20] J. Wang, J. Pu, P. R. Moore and Z. Zhang. "Modelling study and robust control of air motor systems", International Journal of Control, Vol.71, pp459-476, 1998.

[21] Hassan K Khalil, Nonlinear Systems, Prentice - Hall International (UK), 1996.

[22] A.F. Khalil and J.H. Wang, "A New Stability and Time-Delay Tolerance Analysis Approach for Networked Control Systems", The Proceedings of the 49th IEEE Conference on Controlol and Decision, pp. 4753-4758, 2010.

[23] Dong-Sung Kim, Young Sam Lee, Wook Hyun Kwon, and Hong Seong Park, "Maximum Allowable Delay Bounds of Networked Control Systems", Journal of Control Engineering Practice, Vol. 11, 1301-1313, 2003.

[24] Chen Peng, Yu-Chu Tian, and Moses O. Tade, "State Feedback Controller Design of Networked Control Systems with Interval Time-Varying Delay and Nonlinearity", International Journal of Robust and Nonlinear Control", pp. $1-16,2007$. 